\title{
A simple, low-cost staining method for rapid-throughput analysis of tumor spheroids
}

Frank Eckerdt ${ }^{1}$, Angel Alvarez ${ }^{2}$, Jonathan Bell ${ }^{1}$, Constadina Arvanitis $^{3}$, Asneha lqbal1,4, Ahmet D. Arslan ${ }^{1}$, Bo Hu², Shi-Yuan Cheng $^{2}$, Stewart Goldman ${ }^{1,4}$, and Leonidas C. Platanias ${ }^{1,5}$ ${ }^{1}$ Robert H. Lurie Comprehensive Cancer Center and Division of Hematology- Oncology, Northwestern University Feinberg School of Medicine, Chicago, IL, ${ }^{2}$ Department of Neurology, Robert H. Lurie Comprehensive Cancer Center, Northwestern Brain Tumor Institute, Northwestern University Feinberg School of Medicine, Chicago, IL, ${ }^{3}$ Center for Advanced Microscopy and Department of Pathology, Northwestern University Feinberg School of Medicine, Chicago, IL, ${ }^{4}$ Division of Hematology and Oncology, Ann \& Robert H. Lurie Children's Hospital of Chicago, Chicago, IL, and ${ }^{5}$ Department of Medicine, Jesse Brown VA Medical Center, Chicago, IL

BioTechniques 60:43-46 (January 2016) doi 10.2144/000114372

Keywords: neurospheres; tumor spheroids; cancer stem cell; glioblastoma; acridine orange; microscopy

Supplementary material for this article is available at www.BioTechniques.com/article/114372.

Tumor spheroids are becoming an important tool for the investigation of cancer stem cell (CSC) function in tumors; thus, low-cost and highthroughput methods for drug screening of tumor spheroids are needed. Using neurospheres as non-adherent three-dimensional (3-D) cultures, we developed a simple, low-cost acridine orange (AO)-based method that allows for rapid analysis of live neurospheres by fluorescence microscopy in a 96-well format. This assay measures the cross-section area of a spheroid, which corresponds to cell viability. Our novel method allows rapid screening of a panel of anti-proliferative drugs to assess inhibitory effects on the growth of cancer stem cells in 3-D cultures.

Solid tumors grow in a three-dimensional (3-D) spatial conformation, which is not mimicked by two-dimensional (2-D) monolayer cultures. Non-adherent tumor spheroids are commonly used as 3-D in vitro models in cancer research to provide an intermediate between conventional adherent cancer cell cultures and in vivo xenograft models (1). In addition to providing a 3-D model, tumor spheroids represent an important tool for studying and expanding cancer stem cell (CSC) populations derived from patient samples or established cancer cell lines. CSCs represent a challenge for cancer therapy, as they are often resistant to current therapies (2). Thus, CSCs grown as spheroids have become an important tool to investigate drugs for their potential to inhibit therapy-resistant CSC function.
Recently, novel high-throughput methodologies for studying tumor spheroids have been developed using luminescent, colorimetric, or fluorescent viability reagents to study a variety of tumor spheroid functions such as motility and invasion (3), effects of co-culture of different cell types $(4,5)$, and hypoxia (6). However, most microscopic highthroughput analyses relying on fluorescent probes require removal of the probe from the supernatant before microscopy. For instance, when fluorescein diacetate (FDA) is used as a viability dye, the culture medium, which contains esterases from dead cells, needs to be removed because it can result in a high background signal (7). As tumor spheroids are non-adherent floating structures, removal of excess probe from the supernatant is difficult and may compromise tumor spheroid integrity. Additionally, common cell viability reagents can be costly (see Supplementary Table S1).

Here we present a convenient, low-cost method for spheroid analysis using fluorescent probes and microscopy. We used acridine orange (AO), a cellpermeable organic compound that emits light in the red and orange spectrums and has been used before to stain and analyze multicellular spheroids (8). When $A O$ is combined with single-stranded RNA, AO dimers are created, and the $\mathrm{AO}$ emission maximum shifts to red (640 nm) (9). However, when it intercalates into doublestranded DNA, AO retains its monomeric properties, its fluorescence yield and lifetime increase more than 2-fold, and its emission maximum shifts to $525 \mathrm{~nm}$ (within the green spectrum) $(9,10)$. As tumor spheroids are detected by DNA-bound $\mathrm{AO}$ in the green [fluorescein isothiocyanate (FITC)] channel (525 nm), removal of excess probe is not required, making $\mathrm{AO}$ an ideal tool for visualizing non-adherent, floating spheroids. Additionally, AO is very costeffective compared to other dyes. Using our AO-based method, the staining cost for 1000 assays is $\$ 0.007$, which is more than 5000 times lower than that of other dyes (for cost-comparison of dyes used for spheroid analysis, see Supplementary Table S1).

Here we report a new low-cost and effective method for analysis of acridine orange-stained 3-D tumor spheroids by rapid-throughput fluorescence microscopy in a 96-well format. 
Figure 1. Comparison of neurosphere images acquired by bright-field and fluorescence microscopy, and the final analyzed image. U87 neurosphere cells (1000 cells per well) were seeded into round-bottom 96-well plates in $100 \mu \mathrm{l}$ cancer stem cell (CSC) medium. After 2 weeks, neurospheres were stained with acridine orange $(\mathrm{AO})$ by adding $1 \mu \mathrm{l}$ CSC medium containing $10 \mu \mathrm{g} / \mathrm{mL}$ AO for 1 h. Subsequently, neurospheres were subjected to microscopy. The same neurosphere was visualized by bright-field microscopy (A) and using the FITC channel to detect AO-positive neurosphere cells (B). (C) The region of interest (ROI) in red identified by the HCA/JOBS software was used to determine neurosphere cross-section area. Scale bar: $500 \mu \mathrm{m}$.
A

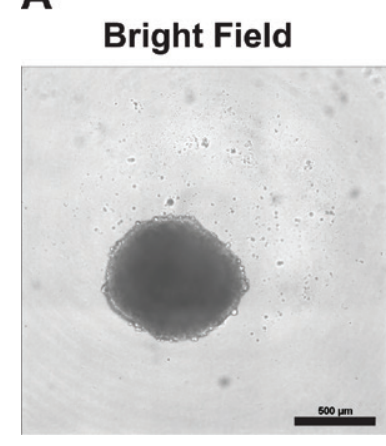

B FITC channel

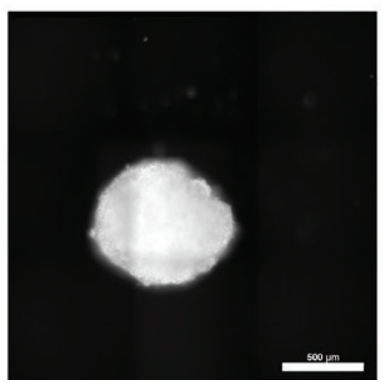

C ROI / cross-section area

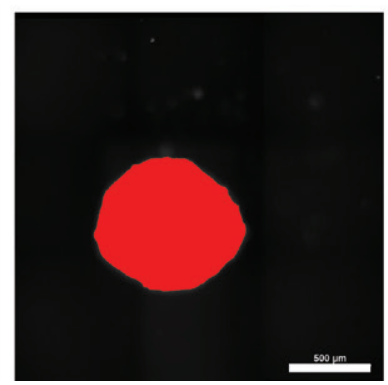

We used neurospheres derived from U87 glioblastoma cells, a well-established model system (11). A detailed protocol can be found in the Supplementary Materials. In brief, adherent U87 cells were dissociated with trypsin and seeded into low-adhesion flasks for suspension culture $\left(4 \times 10^{6}\right.$ cells per $75 \mathrm{~cm}^{2}$ flask) in cancer stem cell medium (CSC medium) comprised of serum-free DMEM/F12 medium supplemented with EGF (20 ng/mL), basic-FGF (20 ng/mL), heparin $(5 \mu \mathrm{g} / \mathrm{mL})$, B27 (2\%), and gentamicin $(0.1 \mathrm{mg} / \mathrm{mL})$. The resulting primary neurospheres were cultured for up to eight passages. U87 neurospheres were then dissociated into single cells and subjected to flow cytometry using a BD FACSAria2 Special Order Research Product (SORP) instrument (BD Biosciences, San Jose, CA) in a biosafety cabinet. Cells were sorted by forward-scattered light (FSC) versus side-scattered light (SSC) and seeded into round-bottom 96-well plates (1000 cells per well in a 96-well suspension culture plate). Seeding cells by flow cytometry allows seeding of exact cell numbers per well while excluding debris or cells from the sub-G1 population, thereby ensuring uniformity at the beginning of the experiment. This is important because uniform spheroids and spheroid sizes are obtained by introducing defined numbers of viable cells to each well, and even small alterations in cell number or viability at seeding can result in substantial differences in neurosphere size after 14 days; however, manual counting and seeding of cells represents a viable alternative that has been used successfully by many laboratories (3-7). U87 neurospheres were allowed to grow for 2 weeks and then stained with $0.1 \mu \mathrm{g} /$ $\mathrm{mL} A O$ for 1 hour. Subsequently, neurospheres were imaged with a Nikon (Melville, NY) Eclipse Ti inverted microscope with an automated stage and a Nikon 10x air PlanApo objective (NA 0.45). Images were
A

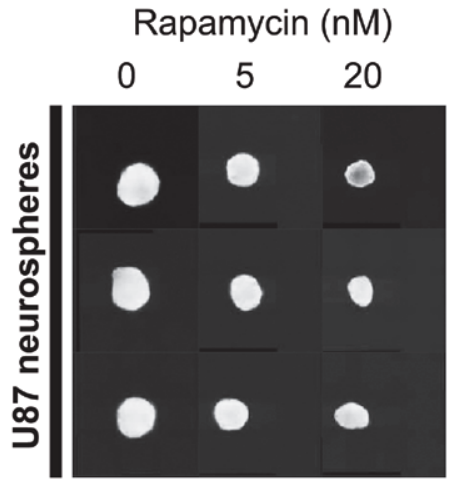

B

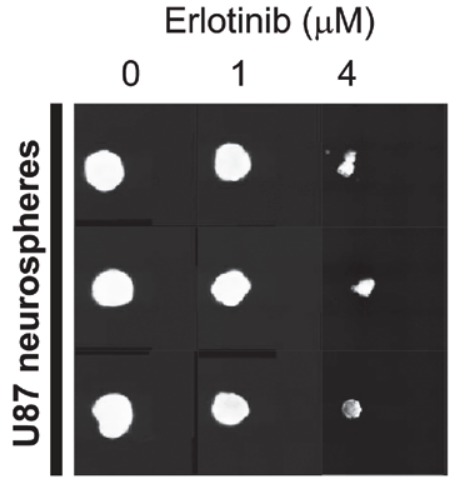

C
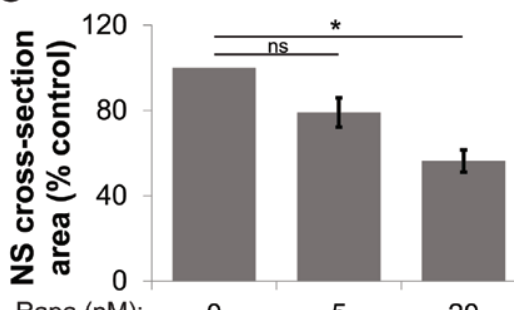

D

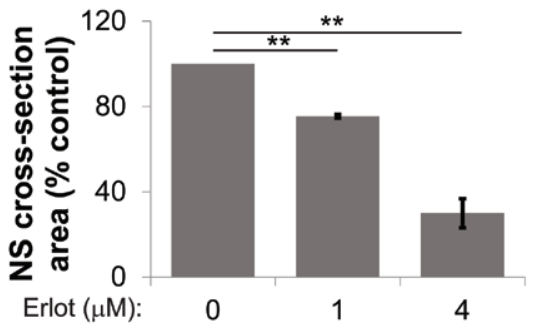

E

$\mathbf{F}$
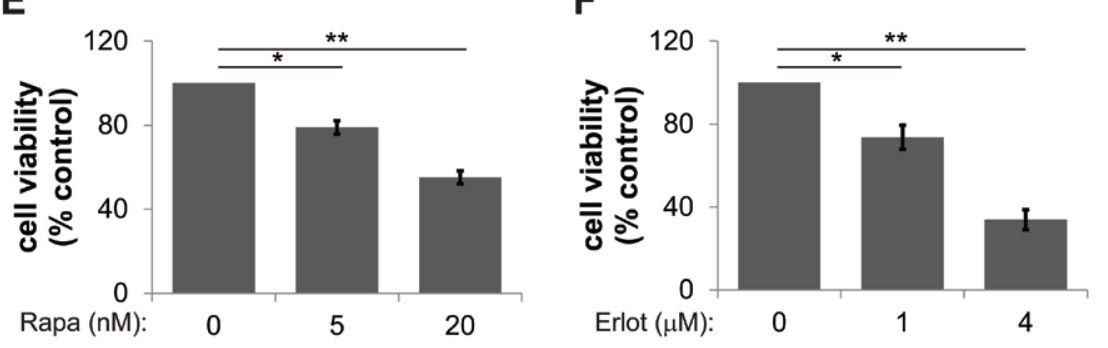

Figure 2. Neurosphere size as measured by spheroid cross-section area corresponds with cell viability. U87 neurosphere cells were seeded into round-bottom 96-well plates (1000 cells per well) and were treated with the indicated concentrations of rapamycin or erlotinib. After 2 weeks, neurospheres were stained with $0.1 \mu \mathrm{g} / \mathrm{mL}$ acridine orange (AO) for $1 \mathrm{~h}$. Cells were analyzed by fluorescence microscopy (FITC channel), followed by measurement of neurosphere cross-section area using the HCA/JOBS software. ( $\mathrm{A}$ and $\mathrm{B}$ ) Tile-view of $\mathrm{AO}$-stained neurospheres (in triplicate) detected in the FITC channel after treatment with DMSO as a control or the indicated concentrations of rapamycin (A) or erlotinib (B) both dissolved in DMSO. (C and D) Neurosphere images taken 2 weeks after incubation with DMSO and the indicated concentrations of rapamycin (C) or erlotinib (D) were analyzed for neurosphere cross-section area as described in the text. Data from 3 independent experiments are shown, each done in 10 technical replicates. Data are expressed as percentages of control DMSO-treated sample values. ( $E$ and F) Neurosphere viability as determined by WST-1 assay after 2 weeks of incubation with DMSO and the indicated concentrations of rapamycin (E) or erlotinib (F). Means \pm SEM are shown from the values of 3 independent experiments, each done in 10 technical replicates. Data are expressed as percentages of DMSO-treated samples (control). ${ }^{*}, P<0.05,{ }^{* *}, P<0.01$ using a paired $t$-test. 
and analyzed data. B.H., S.-Y.C., and S.G. provided reagents, analyzed data, and edited the manuscript. L.C.P. designed studies, analyzed data, and edited the manuscript.

\section{Acknowledgment}

The work was supported by $\mathrm{NIH}$ grants R01 CA121192, CA155566, CA77816 and grant I01CX000916 from the Department of Veterans Affairs (LCP). JB is supported in part by a NIH/NCl training grant, T32 CA09560. This work was also supported by $\mathrm{NIH}$ grants R01 CA158911, R01 CA158911-S3, R01 NS093843, a Zell scholar award from Zell Family Foundation, Northwestern Brain Tumor Institute (SYC) and a Brain Cancer Research Award from James S. McDonnell Foundation $(\mathrm{BH})$. This work was also supported by Northwestern University's Center for Advanced Microscopy and the Flow Cytometry Facility, as well as Cancer Center Support Grant NCl CA060553. This paper is subject to the $\mathrm{NIH}$ Public Access Policy.

\section{Competing interests}

C.A. is supported in part by Nikon. The other authors declare no competing interests.

\section{References}

Weiswald, L.B., D. Bellet, and V. Dangles-Marie. 2015. Spherical cancer models in tumor biology. Neoplasia 17:1-15.

2. Francipane, M.G., J. Chandler, and E. Lagasse. 2013. Cancer Stem Cells: A Moving Target. Curr Pathobiol Rep. 1:111-118.

Vinci, M., S. Gowan, F. Boxall, L. Patterson, M. Zimmermann, W. Court, C. Lomas, M. Mendiola, et al. 2012. Advances in establishment and analysis of three-dimensional tumor spheroidbased functional assays for target validation and drug evaluation. BMC Biol. 10:29.

4. Hoffmann, O.I., C. Ilmberger, S. Magosch, M. Joka, K.W. Jauch, and B. Mayer. 2015. Impact of the spheroid model complexity on drug response. J. Biotechnol. 205:14-23.

Hsiao, A.Y., Y.C. Tung, X. Qu, L.R. Patel, K.J. Pienta, and S. Takayama. 2012. 384 hanging drop arrays give excellent Z-factors and allow versatile formation of co-culture spheroids. Biotechnol. Bioeng. 109:1293-1304.

6. Senkowski, W., X. Zhang, M.H. Olofsson, R. Isacson, U. Hoglund, M. Gustafsson, P. Nygren, S. Linder, et al. 2015. Three-Dimensional Cell Culture-Based Screening Identifies the Anthelmintic Drug Nitazoxanide as a Candidate for Treatment of Colorectal Cancer. Mol. Cancer Ther. 14:1504-1516. Lindhagen, E., P. Nygren, and R. Larsson. 2008. The fluorometric microculture cytotoxicity assay. Nat. Protoc. 3:1364-1369.

8. Johnson, T.S., E. Bain, M.R. Raju, and J.C. Martin. 1980. Correlation of the growth of Chinese hamster V279-17lb multicellular spheroids with cytokinetic parameters. Cytometry 1:65-70.
9. Zelenin, A.V. 1999. Acridine Orange as a Probe for Cell and Molecular Biology, p. 117-135. In W.T. Mason, (Ed.), Fluorescent and Luminescent Probes for Biological Activity: A Practical Guide to Technology for Quantitative Real-Time Analysis (2nd Edition). Academic Press, London, UK.

10. Petit, J.M., M. Denis-Gay, and M.H. Ratinaud. 1993. Assessment of fluorochromes for cellular structure and function studies by flow cytometry. Biol. Cell 78:1-13.

11. Yu, S.C., Y.F. Ping, L. Yi, Z.H. Zhou, J.H. Chen, X.H. Yao, L. Gao, J.M. Wang, and X.W. Bian. 2008. Isolation and characterization of cancer stem cells from a human glioblastoma cell line U87. Cancer Lett. 265:124-134.

12. Feng, H., G.Y. Lopez, C.K. Kim, A. Alvarez, C.G. Duncan, R. Nishikawa, M. Nagane, A.J. Su, et al. 2014. EGFR phosphorylation of DCBLD2 recruits TRAF6 and stimulates AKT-promoted tumorigenesis. J. Clin. Invest. 124:3741-3756.

13. Eckerdt, F., E. Beauchamp, J. Bell, A. Iqbal, B. Su, R. Fukunaga, R.R. Lulla, S. Goldman, and L.C. Platanias. 2014. Regulatory effects of a Mnk2-elF4E feedback loop during mTORC1 targeting of human medulloblastoma cells. Oncotarget. 5:8442-8451.

Received 11 May 2015; accepted 23 September 2015.

Address correspondence to Frank Eckerdt, Robert H. Lurie Comprehensive Cancer Center, Northwestern University Feinberg School of Medicine, 303 East Superior Street, Lurie 3-220, Chicago, IL 60611. E-mail: frank.eckerdt@northwestern.edu

To purchase reprints of this article, contact: biotechniques@fosterprinting.com

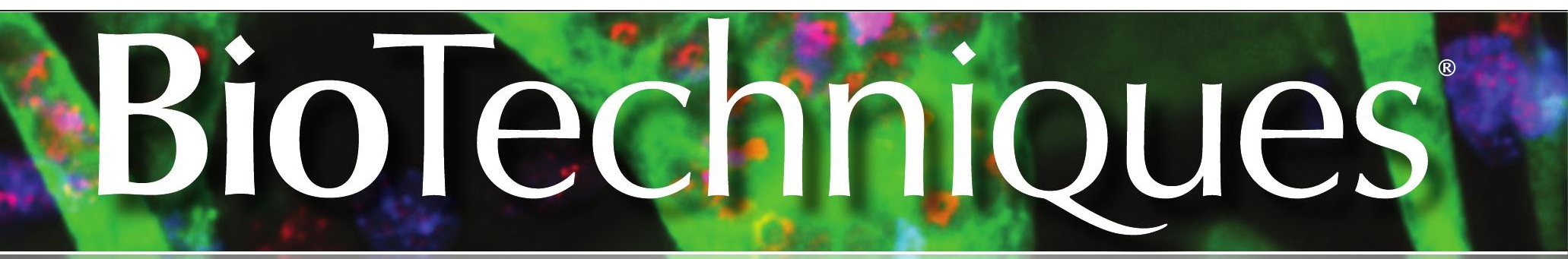

\section{The Bjotechniques Vilobile App}

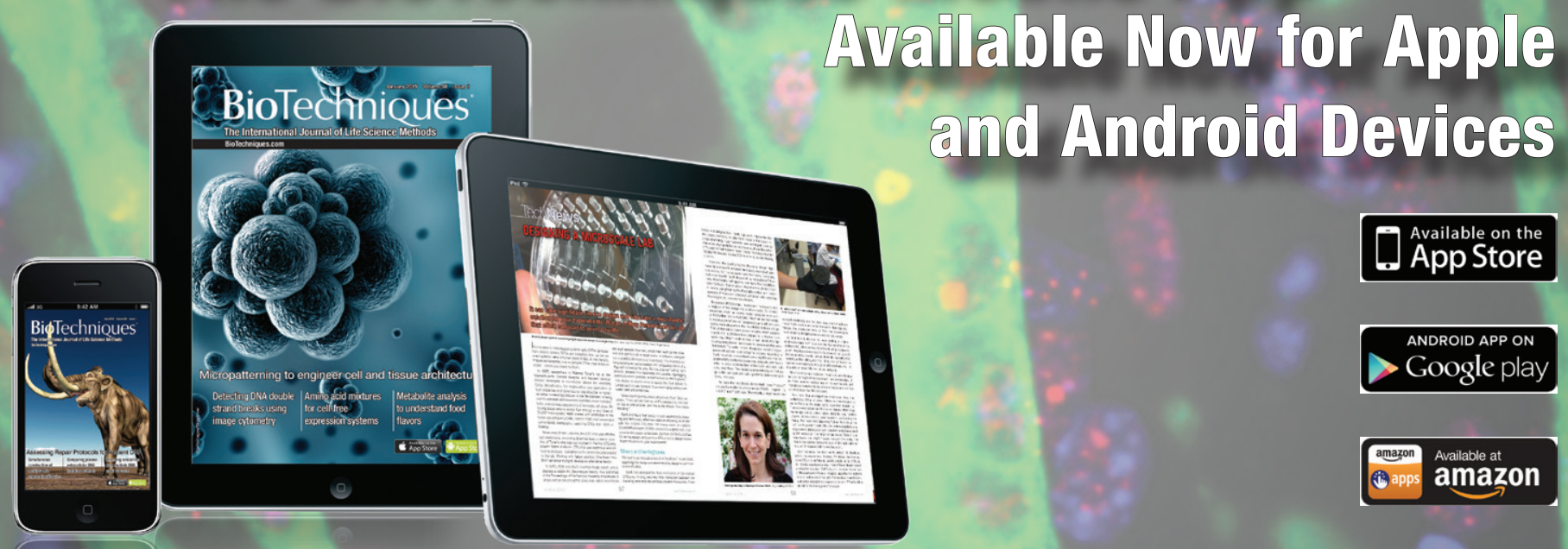

Visit BioTechniques.com/Digital to learn more and BioTechniques.com/Subscribe to receive monthly issue alerts. 